Tobias F. Jakobs

Christoph R. Becker

Bernd Ohnesorge

Thomas Flohr

Christoph Suess

Uwe J. Schoepf

Maximilian F. Reiser

\section{Multislice helical CT of the heart with retrospective ECG gating: reduction of radiation exposure by ECG-controlled tube current modulation}

noise and were tested for significant differences. There was no significant difference between group 1 and group 2 with respect to image noise. Radiation exposure with and without tube current modulation was 1.0 and $1.9 \mathrm{mSv}(p<0.0001)$, respectively, for males and 1.4 and $2.5 \mathrm{mSv}$ $(p<0.0001)$, respectively, for females; thus, there was a mean dose reduction of $48 \%$ for males and $45 \%$ for females, respectively. The ECG-controlled tube current modulation allows significant dose reduction when performing retrospectively ECG-gated MSCT of the heart.

Keywords Multislice helical CT . Coronary artery calcification . Radiation exposure $\cdot$ Retrospective ECG gating $\cdot$ Dose reduction

\section{Introduction}

Cardiovascular diseases are the most common causes of premature death in developed countries. A major fraction is attributable to coronary artery disease which may result in sudden cardiac death [1]. In approximately 50\% of patients myocardial infarction is the first sign of coronary atherosclerosis and more than $50 \%$ of patients suffering an acute myocardial event die within the first month after onset of the acute phase [2]. A reduction of mortality caused by myocardial infarction may be achieved if coronary atherosclerosis can be detected at an early stage of the disease before symptoms occur; therefore, there is need for an effective tool that allows identification of patients at increased risk for future cardiac events.
The independent predictive value of coronary calcifications in predicting cardiac events is currently under investigation in large trials [3, 4]. Further indications for detection and quantification of coronary calcifications may consist of investigating symptomatic patients with atypical chest pain [5], and of following the progression of coronary atherosclerosis after risk factor modification or medical treatment $[6,7]$. Both, electron beam CT and conventional single detector row CT, have been used for detection and quantification of coronary calcifications as a sign of coronary atherosclerosis [8].

New-generation CT systems with four-detector arrays and a minimum rotation time of $500 \mathrm{~ms}$ (Somatom VolumeZoom, Siemens, Forchheim, Germany) were introduced into clinical routine in 1998. Continuous helical scanning, 500-ms rotation time, simultaneous acquisition 
of four slices and simultaneous recording of the ECG signal allow for retrospectively ECG-gated reconstruction of image data. Retrospectively ECG-gated helical scanning is capable of covering the entire coronary tree without gaps within a single breath-hold period for detection and quantification of coronary artery calcification [9]. Dedicated cardiac reconstruction algorithms are in use providing a minimum temporal resolution of $125 \mathrm{~ms}$ for phase-selective reconstruction in the diastolic phase to minimize cardiac motion artifacts [10].

Traditionally, prospective ECG triggering with sequential slice-by-slice acquisition was used for quantification of coronary calcium. The radiation exposure to the investigated individual with this technique was determined at 0.8 and $0.5 \mathrm{mSv}$ with use of electron beam CT and MSCT, respectively, when adjusted for image quality [11]; however, the drawback of a protocol involving prospective triggering may be reduced reproducibility compared with a continuous helical acquisition of the coronary artery tree that allows for shorter acquisition time, overlapping slice reconstruction and thus reduced partial-volume effects [12].

However, retrospective helical acquisition requires slow table movement during scanning for oversampling projection data for gap-less and heart-phase-consistent reconstruction. If images are to be reconstructed in the diastolic phase, only this kind of protocol will lead to a significant amount of redundant radiation exposure. Because the detection and quantification of coronary artery calcification is considered to be a screening tool in potentially healthy people, radiation exposure should be as low as possible.

The purpose of our study was to evaluate the potential of ECG-controlled tube current modulation ("ECG pulsing") with retrospectively ECG-gated cardiac CT and to determine whether this technique allows for reduction of radiation exposure [13] to the investigated individual.
Table 1 Comparison of group 1 (standard protocol) and group 2 (tube current modulation)

\begin{tabular}{lclc}
\hline & Group 1 & Group 2 & $p$-value \\
\hline No. of patients & 50 & 50 & - \\
Females & 14 & 14 & - \\
Mean age & 55.7 & 56.3 & 0.8002 \\
Mean BMI & 25.59 & 25.65 & 0.9382 \\
Mean heart rate & 70 & 64 & 0.0077 \\
Mean SD & 21.3 & 21.1 & 0.8262 \\
Mean mAs & 100 & 53.3 & $<0.0001$ \\
Effective dose (mSv, male) & 1.95 & 1.03 & $<0.0001$ \\
Effective dose (mSv, female) & 2.48 & 1.37 & $<0.0001$ \\
CTDI $_{\text {Weff }}$ (mGy) & 9.4 & 5.1 & - \\
\hline
\end{tabular}

\section{Materials and methods}

Patients

A total of 100 patients with suspicion of coronary artery disease referred by the Department of Cardiology were included in this study. Since these patients underwent this examination as part of their clinical work-up for coronary artery disease (CAD), and data were analysed in a retrospective manner, institutional approval was not sought.

The first group of 50 consecutive patients (group 1, 14 women, 36 men, mean age 55.7 \pm 12.05 years) was investigated before ECGcontrolled tube current modulation became available. After installation the second group of another 50 consecutive patients (group 2 , 14 women, 36 men, mean age $56.3 \pm 11.6$ years) was investigated with automated ECG-controlled tube current modulation.

For obvious ethical reasons, we did not expose patients twice with and without tube current modulation. Patients were investigated consecutively without randomization. As image noise depends on the volume in the scan field, patient data necessary to calculate the body mass index (BMI) were recorded in each patient to compare image noise levels relative to BMI in the two groups. The heart rate was documented in each patient. Patient group characteristics are summarized in Table 1. Patients with arrhythmia were excluded from the study.
Fig. 1 Tube current modulation. Retrospectively ECG-gated four-slice spiral scanning with prospectively ECG-controlled tube current modulation for reduced radiation exposure. Tube current is at nominal value (time interval is $\Delta T_{N}$ during diastole and is reduced by $80 \%$ during phases of high cardiac motion)

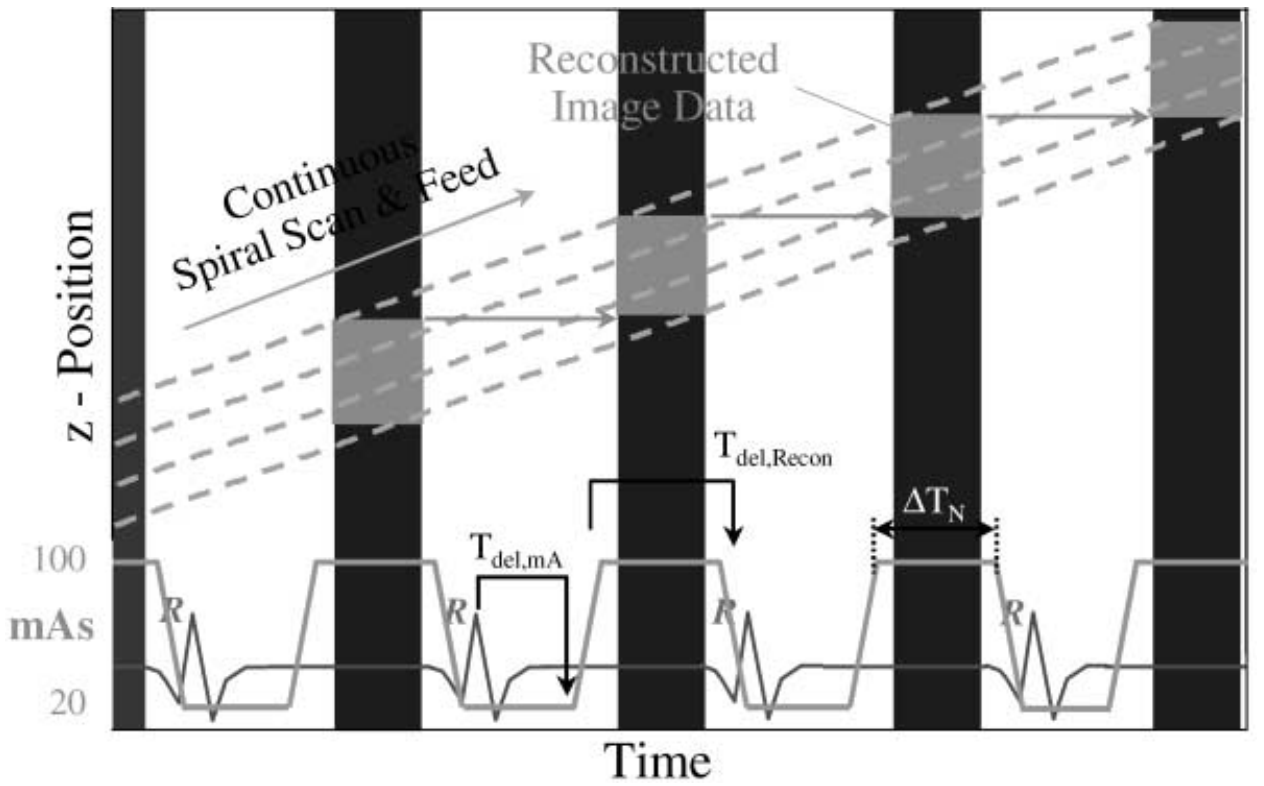




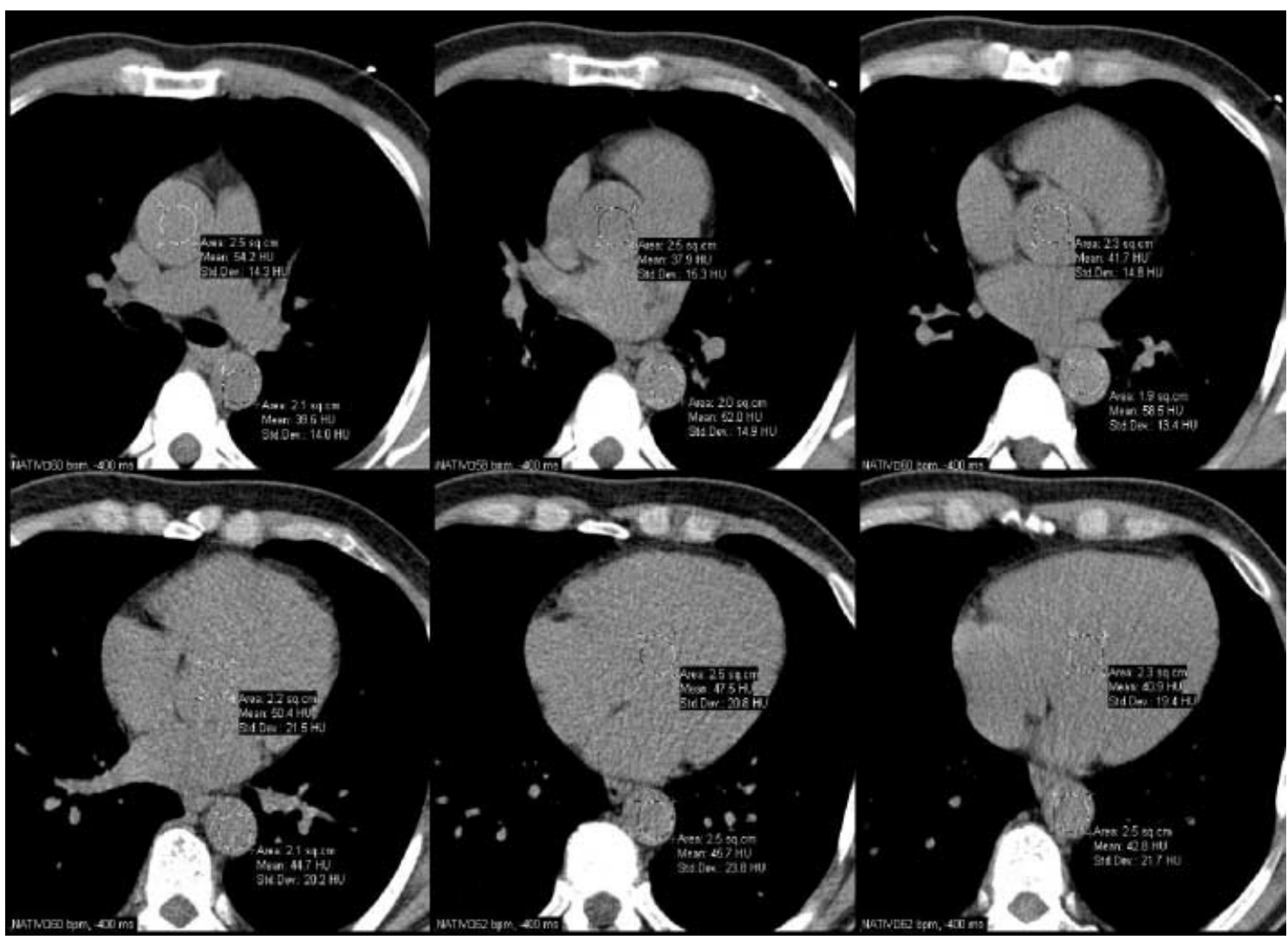

Fig. 2 Acquisition of CT numbers and standard deviations. A total of 12 regions of interest every $1.5 \mathrm{~cm}$ in $\mathrm{z}$-direction were placed in every set of patient images. Half of them were placed in a posterior column in the area of the descending aorta, the other half were placed in a more anterior column along the aortic arch and the left ventricle

\section{MSCT scanning and ECG-gating technique}

For scanning the coronary arteries for detection and quantification of coronary calcifications a newest generation MSCT scanner (Somatom VolumeZoom, Siemens, Forchheim, Germany) was used. The range of the heart $(120 \mathrm{~mm})$ was covered in one breathhold period of $15-\mathrm{s}$. Table feed was $7.5-\mathrm{mm} / \mathrm{s}$ (helical pitch 1.5). The nominal scanning parameters were set to $120 \mathrm{kV}, 75 \mathrm{~mA}$ (=100 mAs effective), $500-\mathrm{ms}$ rotation time using a $4 \times 2.5-\mathrm{mm}$ collimation. These parameters were applied throughout the entire scan without dose modulation in the first group of 50 consecutive patients. In the second group of patients these nominal scan parameters were used in conjunction with ECG-controlled tube current modulation.

For ECG-controlled tube current modulation (ECG pulsing), the tube output is raised to the nominal level within every cardiac cycle during a limited interval in the diastolic phase where data are most likely to be reconstructed. During the remaining part of the cardiac cycle the tube output can be reduced by approximately $80 \%$ by a corresponding decrease of the tube current (Fig. 1).

Continuous volume reconstruction is still possible in all phases of the cardiac cycle. The width of the time interval $\Delta T_{N}$ with nominal tube output during diastole can be selected in a way that allows shifting of the ECG-gated reconstruction interval with individual adjustment to the patient's ECG to reconstruct during the phase of least cardiac motion. Additionally, a predefined overlap of $\Delta T_{N}$ with the window of temporal resolution (i.e. 125-250 ms) can compensate for inconsistent prospectively ECG-controlled timing of the nominal tube output that may occur due to moderate heart rate changes during the scan (e.g. Valsalva manoeuvre).

In a pilot study (data not shown) the best trade-off between reduction of radiation exposure and flexibility of ECG gating was found with a fix time interval $\Delta T_{N}=400 \mathrm{~ms}$ that allows for a 150 -ms shift of a 250-ms temporal resolution window without loss of data quality. The tube current adaptation, the function of tube current over time, forms a trapezoidal curve. The relative position of the windows of nominal tube output within the heart cycles was defined prior to the scan (475-75 ms prior to the R-wave) via a preliminary estimate of the optimal reconstruction interval (e.g. $400 \mathrm{~ms}$ prior to the R-wave) based on the individual ECG. During the scan the delay time between a detected R-wave and the onset of the tube current increase was prospectively calculated based on the average of the three prior RR intervals.

After scanning was completed all images were reconstructed during diastole at $400 \mathrm{~ms}$ prior to the R-wave of the ECG with a medium smooth reconstruction kernel ("B35f", $50 \%$ value of the modulation transfer function $\approx 4.0 \mathrm{~cm}^{-1}$ ) every $1.5 \mathrm{~mm}$ through the entire data set.

The effective milliamperes as recorded in the image data were used to calculate the effective dose according to ICRP60 [14] using commercially available software (WinDose2.0a, Scanditronix Wellhofer, Bartlett, Tenn.)

\section{Statistical evaluation}

The reconstructed CT images were evaluated on an external workstation (Magic View 1000, Siemens, Forchheim, Germany). A total of 12 regions of interest (ROIs, area $2.5 \mathrm{~cm}^{2}$ ) every $1.5 \mathrm{~cm}$ in z-direction were placed in every set of patient images (Fig. 2). Half of them were placed in a posterior column in the area of the descending aorta, the other half were placed in a more anterior column 


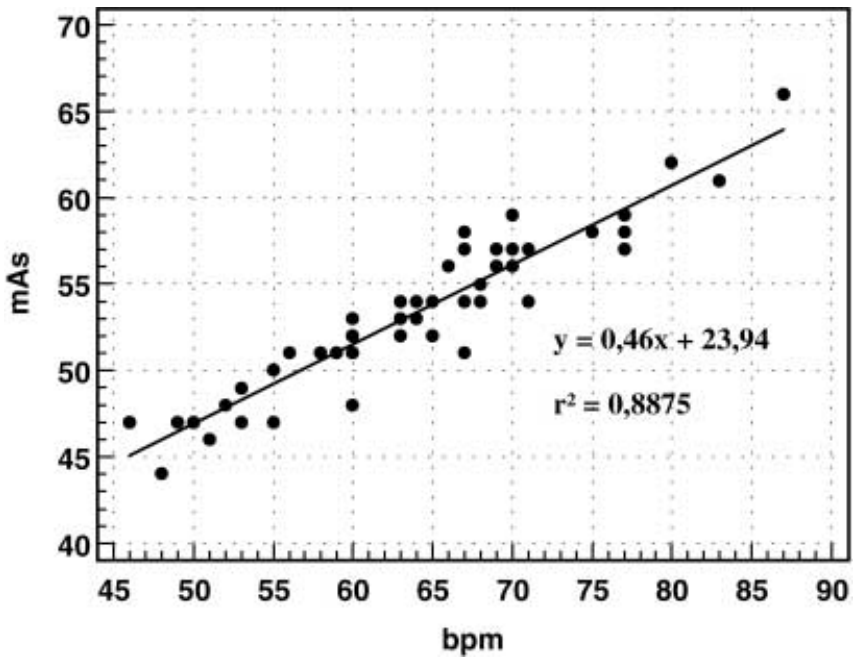

Fig. 3 Heart rate depending dose reduction. Regression plot illustrates relation between heart rate and radiation exposure $\left(y=0.46 x+23.94, r^{2}=0.8875\right)$ and demonstrates that the tube current and therefore the radiation exposure is more reduced in patients with low heart rates than in those with higher heart rates

along the aortic arch and the left ventricle. The mean of the CT number and the standard deviation were determined for each of these 12 ROIs. For statistical evaluation an unpaired $t$-test was used. A $p$-value $<0.01$ was considered to indicate a significant difference.

\section{Results}

Owing to the short breath-hold time the examination was successfully completed in every patient. In the patients who underwent coronary artery screening using the standard protocol without tube current modulation (group 1) the mean heart rate varied between 38 and 103 beats per minute (mean $70 \pm 13$ beats per minute $=\mathrm{bpm}$, median 68 ). The BMI varied between 16.9 and 34.9 (mean 25.59 \pm 3.6 ).

In the patients who underwent the alternative protocol for dose reduction the mean heart rate varied between 46 and 87 beats per minute (mean $64 \pm 9$ bpm, median $64 \mathrm{bpm}$ ), and the mean BMI was 25.65 \pm 3.4 (min 20.4, max 37.2). Average BMI for groups 1 and 2 were not significantly different (25.59 and 25.65, respectively; $p=0.938$ ).

A mean noise level was calculated by the mean of the standard deviation of all 600 ROIs per group that were assessed. The mean noise level was not significantly different between these two groups $(21.3$ and $21.1 \mathrm{HU}$, respectively; $p=0.826$ ).

The mean tube current for groups 1 and 2 was 100 and $53 \mathrm{mAs}$, respectively. When calculating the effective dose using these parameters male and female patients in group 1 received an effective dose of 1.9 and $2.5 \mathrm{mSv}$, respectively. In group 2 (with tube current modulation) effective dose was significantly lower, for male and female patients with 1.0 and $1.4 \mathrm{mSv}$, respectively. This corresponds to a mean reduction of effective radiation dose of $45 \%$ for females and $48 \%$ for males, respectively, without a significant change of the image noise level.

When heart rate and applied radiation dose were correlated (Fig. 3) it was shown that a higher heart rate was associated with a higher radiation dose applied to the patient $(60 \mathrm{bpm} \approx 50 \mathrm{mAs}, 70 \mathrm{bpm} \approx 55 \mathrm{mAs}$, $80 \mathrm{bpm} \approx 62 \mathrm{mAs}$ ).

\section{Discussion and conclusion}

Computed tomography is the most important source of ionizing radiation for the general population. Concern has been raised over the perceived increase in radiation dose associated with the advent of advanced CT techniques. Novel developments in CT technique, such as MSCT, allow for a host of advanced applications such as cardiac imaging. Used as a tool in the realm of preventive care, cardiac CT is increasingly performed in the population of asymptomatic, a priori healthy individuals, where use of excessive ionizing radiation is of special concern. Our study aimed at finding means to reduce the radiation burden associated with state-of-the-art cardiac CT imaging.

Reconstructed images acquired by using the protocol with tube current modulation did not result in a measurable increase in image noise, but a mean dose reduction of $47 \%$. Due to the design of our study which avoided intraindividual comparisons with double exposure of patients, we could not directly evaluate the effect of ECG-controlled tube modulation on the detection and quantification of coronary artery calcification; however, it can be assumed that in images with comparable noise level the sensitivity and accuracy for the detection of coronary calcification will remain unchanged.

Based on our initial results, we believe that ECG-controlled tube current modulation should be generally recommended for use with retrospectively ECG-gated helical scanning for the detection and quantification of coronary calcifications.

The relative radiation reduction that can be achieved with ECG-controlled tube modulation will be proportionally higher for studies acquired with thinner slices (e.g. $1.25 \mathrm{~mm})$. Based on the currently used scan parameters for CT angiography of the heart $(120 \mathrm{kV}, 300 \mathrm{~mA}$, $4 \times 1-\mathrm{mm}$ slice collimation, feed $3 \mathrm{~mm} / \mathrm{s}$ ), we calculated an effective dose of 7.1 and $9.6 \mathrm{mSv}$ for male and female patients, respectively, to cover a $120-\mathrm{mm}$ scan range. Accordingly, the radiation dose associated with a thin-slice protocol can potentially be reduced to 3.8 and $5.1 \mathrm{mSv}$, respectively, assuming a mean reduction of approximately $47 \%$ with ECG-controlled tube current modulation as demonstrated in the current study. The CT angiography of the heart will then be within the range of a conventional diagnostic coronary angiography procedure [11]. 
In our study only patients with a regular sinus rhythm were included. If substantial arrhythmia occurs during the scan, prospective control of the tube current and retrospective positioning of the reconstruction intervals may not perfectly match. In this case, increased image noise may occur in some of the reconstructed images; however, a well-defined overlap of $\Delta T_{N}$ with the window of temporal resolution $(250 \mathrm{~ms})$ is able to compensate for inconsistent prospectively ECG-controlled timing of the nominal tube output which may occur due to moderate heart rate changes during the scan (e.g. Valsalva manoeuvre). Further studies are needed to investigate to what degree such a mismatch and the resulting increase in image noise influence the diagnostic quality and impact the detection and quantification of coronary artery calcifications in patients with arrhythmia.

Since the tube output is reduced during phases with high cardiac motion (i.e. systolic phase) but not completely suspended, it needs to be investigated if continuous volume reconstruction remains possible in all phases of the cardiac cycle. In particular, functional imaging that relies on image reconstruction during different heart phases might be still feasible, as this application does not require thin-slice reconstruction. For image reconstruction during phases of reduced tube output, an appropriate signal-to-noise ratio can probably be maintained by primary or secondary reconstruction of thicker slices.

The proportion of radiation reduction is higher in patients with slow heart rates than in those with faster heart rates (Fig. 3). For retrospectively ECG-gated helical CT of the heart continuous volume coverage is achieved by adjusting the helical pitch to the patient's heart rate [11]. Use of different pitch values adjusted to the minimum heart rate of the patient thus results in different radiation doses for patients with different heart rates [15]. We used a pitch of 1.5 to investigate patients with a heart rate of 40 and above. In patients with higher heart rates, the pitch can be increased for further reduction of the radiation dose; thus, using pitch values optimally adapted to the heart rate of the individual patient may increase the beneficial effect of prospective ECG-modulated tube current in patients with higher heart rates.

It is well known that mathematical estimations of effective doses for CT protocols may underestimate as well as overestimate the radiation exposure to the patient [11]. Inhomogeneous dose distribution in cardiac CT, which is likely to occur, may not be reflected in a static mathematical formula. Our results indicate that prospectively ECG-controlled tube current modulation significantly reduces radiation exposure without effecting image quality; therefore, this technique should be used with retrospective ECG-gated cardiac CT examinations in patients with regular sinus rhythm. We have determined similar signal-to-noise ratios in both groups; therefore, we do not expect a significant impact on detection of coronary artery calcifications. Further investigations will be necessary to determine reliability and reproducibility of this technique.

\section{References}

1. Friedewald ST, Wyngaarden JB, Smith LH (eds) (1988) Cecil's textbook of medicine, 18th edn. Saunders, Philadelphia, pp 179-183

2. Tunstall-Pedoe H, Kuulasmaa K, Mahonen M, Tolonen H, Ruokokoski E, Amouyel P (1999) Contribution of trends in survival and coronary event rates to changes in coronary heart disease mortality: 10-year results from 37 WHO MONICA project populations. Monitoring trends and determinants in cardiovascular disease. Lancet 353:1547-1557

3. O'Malley P, Taylor A, Gibbons R et al. (1999) Rationale and design of the prospective army coronary calcium (PACC) study: utility of electron beam computed tomography as a screening test for coronary artery disease and as an intervention for risk factor modification amoung young, asymptomatic, active-duty United States Army personal. Am Heart J 137:932-941
4. National Institutes of Health. NHLBI launches 10-year study on early detection of heart disease: National Heart, Lung and Blood Institute, 2000, http://www.nhlbi.nih.gov/new/press/ sep14-00.htm

5. Laudon DA, Vukov LF, Breen JF, Rumberger JA, Wollan PC, Sheedy PF (1999) Use of electron-beam computed tomography in the evaluation of chest pain patients in the emergency department. Ann Emerg Med 33:15-21

6. Wexler L, Brundage B, Crouse J et al. (1996) Coronary artery calcification: pathophysiology, epidemiology, imaging methods and clinical implications. A statement for health professionals from the American Heart Association. Writing Group. Circulation 94:1175-1192

7. Callister TQ, Raggi P, Cooli B, Lippolis NJ, Russo DJ (1998) Effect of HMG-CoA reductase inhibitors on coronary artery disease as assessed by electron-beam computed tomography. N Engl J Med 339:1972-1978
8. Becker C, Jakobs T, Aydemir S et al. (2000) Helical and single slice conventional versus electron beam CT for quantification of coronary artery calcification. Am J Roentgenol 174:543-547

9. Becker C, Kleffel T, Crispin A et al. (2001) Coronary artery calcium measurement: agreement of multirow detector and electron beam CT. Am J Roentgenol 176:1295-1298

10. Ohnesorge B, Flohr T, Becker CR et al. (2000) Cardiac imaging by means of electrocardiographically gated multisection spiral CT: initial experience. Radiology 217:564-571

11. Becker C, Schätzl M, Feist H et al. (1999) Assessment of the effective doese for routine protocols in conventional CT, electron beam CT and coronary angiography. Rofo Fortschr Geb Röntgenstr Neuen Bildgeb Verfahr 170:90-104 [in German] 
12. Ohnesorge B, Becker CR, Kopp AF, Fischbach R, Knez A, Flohr T (2000) Reproducibility and consistency of volumetric coronary calcium scoring with ECG-gated multislice spiral CT.

Radiology 217:233
13. Ohnesorge B, Flohr T, Becker CR, Kopp AF, Knez A, Reiser MF (2000) Dose evaluation and dose reduction strategies for ECG-gated multislice spiral CT of the heart. Radiology 217:487

14. International Commission on Radiation Protection (1990) Recommendation of the ICRP. ICRP Publication 60. ICRP Publ 60. Pergamon Press, Oxford
15. Klingenbeck K, Schaller S, Flohr T, Ohnesorge B, Kopp AF, Baum U (1999) Subsecond multislice computed tomography: basics and applications. Eur J Radiol 31:110-124 